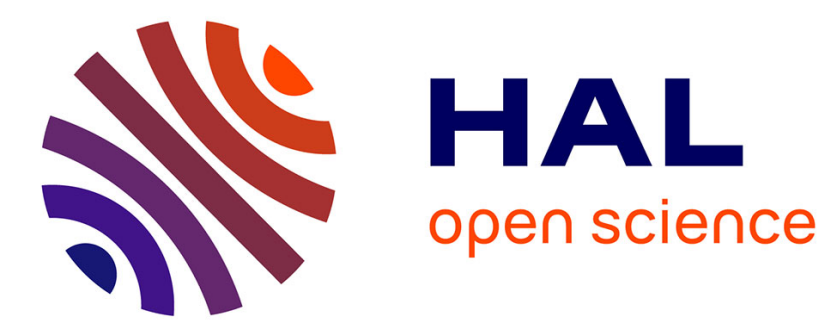

\title{
Ultrasonic Studies of Incommensurate Phase Transitions
} V. Samulionis, V. Valevicius, J. Banys, A. Brilingas

\section{To cite this version:}

V. Samulionis, V. Valevicius, J. Banys, A. Brilingas. Ultrasonic Studies of Incommensurate Phase Transitions. Journal de Physique IV Proceedings, 1996, 06 (C8), pp.C8-405-C8-408. 10.1051/jp4:1996887 . jpa-00254698

\section{HAL Id: jpa-00254698 https://hal.science/jpa-00254698}

Submitted on 1 Jan 1996

HAL is a multi-disciplinary open access archive for the deposit and dissemination of scientific research documents, whether they are published or not. The documents may come from teaching and research institutions in France or abroad, or from public or private research centers.
L'archive ouverte pluridisciplinaire HAL, est destinée au dépôt et à la diffusion de documents scientifiques de niveau recherche, publiés ou non, émanant des établissements d'enseignement et de recherche français ou étrangers, des laboratoires publics ou privés. 


\title{
Ultrasonic Studies of Incommensurate Phase Transitions
}

\author{
V. Samulionis, V. Valevicius, J. Banys and A. Brilingas \\ Department of Physics, Vilnius University, Sauletekio al. 9/3, 2054 Vilnius, Lithuania
}

\begin{abstract}
The temperature dependencies of the ultrasonic velocities and attenuation coefficients are studied in the vicinity of the incommensurate phase transitions of BCCD and AHSe crystals. The largest contribution to the longitudinal ultrasonic anomalies is due to the coupling to the amplitude mode, whose relaxation time $\tau$ diverges as the transition temperature $T_{i}$ is approached with the temperature dependence given by: $\tau=\tau_{0} T_{i} /\left(T-T_{i}\right)$. The amplitudon relaxation time for AHSe crystal $\left(\tau_{0}=5.1 \cdot 10^{-12} \mathrm{~s}\right)$ was found to be considerably longer than for BCCD $\left(\tau_{0}=1.3 \cdot 10^{-13} \mathrm{~s}\right)$. From the measurements of shear ultrasonic wave velocity in incommensurate phase below $T_{i}=$ $164 \mathrm{~K}$ the critical exponent that describes the order parameter temperature behaviour has been calculated for $B C C D$. The value $\beta=0.345$ is consistent with three dimensional $X-Y$ model. The substitution of $\mathrm{Cl}$ by $\mathrm{Br}$ in $\mathrm{BCC}_{1-\mathrm{x}} \mathrm{Br}_{\mathrm{x}} \mathrm{D}$ crystals with $\mathrm{x}<0.24$ manifests itself in smearing of the elastic anomalies and shifting $\mathrm{T}_{\mathrm{i}}$ to lower temperatures with increase of $\mathrm{Br}$ concentration.
\end{abstract}

\section{INTRODUCTION}

From the physical point of view the anomalous behaviour of elastic properties near the phase transitions in various materials is interesting and has been widely studied [1,2]. In ferroelectric crystals the character of elastic anomalies in ultrasonic frequency range $10-100 \mathrm{MHz}$ is strongly depended on the order parameter (polarisation) relaxation time [3]. The polarisation relaxation approach can be used to explain frequency dependent elastic anomalies in ferroelectrics with incommensurate phases below the lock-in transition point $T_{C}$. But below the phase transition from normal to incommensurate phase $T_{i}$ all the acoustic anomalies arise due to the coupling between ultrasonic wave and the so called amplitudon and phason branches of the soft mode. Just below $T_{i}$ the amplitudon mode is most important. The increase of the amplitudon relaxation time must manifest itself in temperature and frequency dependent anomalies of the elastic properties. The amplitudon relaxation time can be different for various materials. As in the case of ferroelectric materials [3], this will lead to the different ultrasonic behaviour. If the relaxation time is relatively short the velocity dispersion must appear in a very narrow temperature region close to $T_{i}$, which is difficult to resolve experimentally. Contrary, if the relaxation time is long, the velocity dispersion can be easily observed at ultrasonic frequencies.

These two cases of anomalous ultrasonic behaviour are described in this contribution. For betaine calcium chloride dihydrate (BCCD) single crystals the velocity dispersion in the frequency range $10-90$ $\mathrm{MHz}$ is negligible at $\mathbf{T}_{i}=164 \mathrm{~K}$. In ammonium hydroselenate (AHSe) a pronounced velocity dispersion near $T_{i}=260.6 \mathrm{~K}$ is observed, showing that the amplitudon relaxation time is long enough in this crystal.

\section{EXPERIMENTAL DETAILS}

The ultrasonic velocity and attenuation measurements were performed using the pulse echo method [4]. In addition, a digital scope and computer processing of amplitude and delay time measurements were employed, what allowed to increase the velocity and attenuation resolution considerably. For excitation 
and detection of longitudinal and shear ultrasonic waves, $\mathrm{LiNbO}_{3}$ transducers of corresponding cuts, vibrating on the main $10 \mathrm{MHz}$ frequency and on harmonics were used. The crystalline samples were prepared so, that both parallel polished faces were perpendicular to the desired crystallographic axis. Silicon oil and silicon grease were used as acoustic bonds for longitudinal and shear waves respectively. The temperature was stabilised and measured to within $0.02 \mathrm{~K}$ accuracy. The estimated ultrasonic velocity and attenuation resolution was better than $10^{-5}$ and $0.2 \mathrm{~dB} / \mathrm{cm}$ correspondingly.

\section{RESULTS AND DISCUSSION}

The temperature dependencies of longitudinal ultrasonic velocity $v$ and attenuation $\alpha$ along $a, b, c$ crystallographic directions of orthorhombic symmetry of BCCD were measured in frequency range $10-90 \mathrm{MHz}$. Large anomalies in $v=f(\mathrm{~T})$ and $\alpha=f(\mathrm{~T})$ were observed at the transition from normal $(\mathrm{N})$ to incommensurate

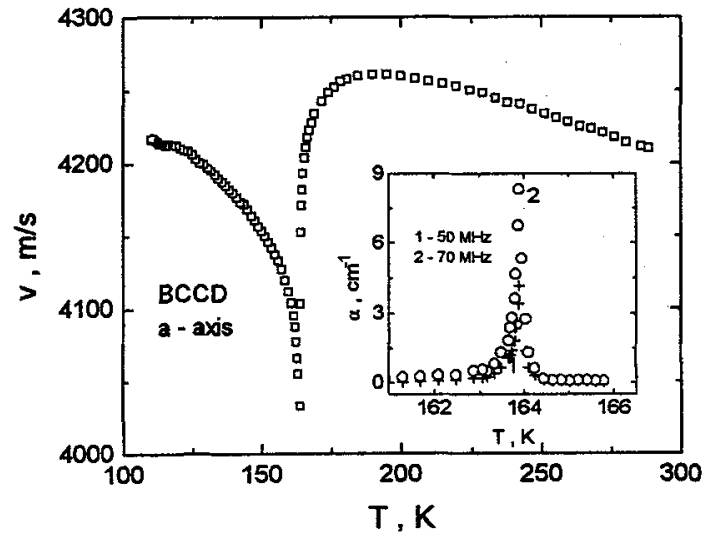

Figure 1: The temperature dependence of longitudinal ultrasonic velocity in BCCD. In the inset: the temperature dependence of attenuation coefficient.

(IC) phase at $T_{i} \cong 164 \mathrm{~K}$. For all directions the longitudinal elastic behaviour was similar. Near $T_{i}$ sharp velocity dips of $5-6 \%$ occurred. In Fig. 1 the $v=f(\mathrm{~T})$, measured along $a$ axis at $f=10 \mathrm{MHz}$ is shown. The data obtained for higher frequencies were identical to $10 \mathrm{MHz}$ measurements. The velocity anomaly is accompanied by sharp ultrasonic attenuation peaks (see the inset in Fig. 1). The attenuation is so high that at $f>70 \mathrm{MHz}$ the received acoustic signal almost disappeared at $T_{i}$, At $f<30 \mathrm{MHz}$ the $\alpha$ anomaly was so narrow that it was difficult to obtain reliable results due to the thermal inhomogenities in the sample. In the IC phase the attenuation coefficient can be described according the relaxation formula [5]:

$$
\alpha=\frac{\Delta v}{v^{2}} \cdot \frac{\omega^{2} \tau}{1+\omega^{2} \tau^{2}},
$$

where $\Delta v$ is the magnitude of the downward step of ultrasonic velocity at $T_{i}$. As it was mentioned above, we could not detect any velocity dispersion, therefore the condition $\omega \tau<1$ must be valid at temperatures close to $\mathrm{T}_{\mathbf{i}}$. In this case Eq. (1) can be simplified to: $\alpha \approx\left(\Delta v / v^{2}\right) \omega^{2} \tau$. The best fits were obtained using $\tau$ written in the form $\tau=\tau_{0} \mathrm{~T}_{\mathrm{i}} /\left(\mathrm{T}_{\mathrm{i}}-\mathrm{T}\right)$ for 50 and $70 \mathrm{MHz}$. frequencies. The result $\tau_{0}=(1.3 \pm 0.1) 10^{-13} \mathrm{~s}$ shows

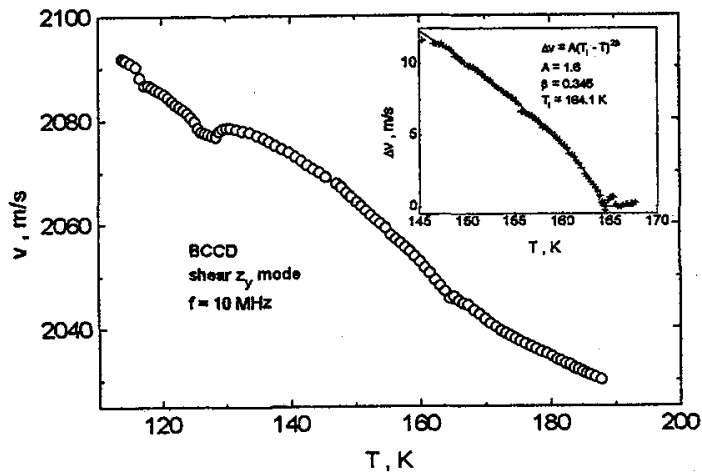

Figure 2: The temperature dependence of shear ultrasonic velocity in $\mathrm{BCCD}$ crystal. In the inset: the temperature dependence of contribution to velocity in IC phase (full line least square fit). that the relaxation time of the order parameter $Q$ (which is amplitudon in IC phase near $T_{i}[6]$ ) is small. The point group of symmetry $D_{2 h}{ }^{16}$ does not allow coupling of shear ultrasonic wave with amplitudon in BCCD crystals. Really, for shear waves there is no softening of ultrasonic velocity near $T_{i}=164 K$ (Fig.2). In this case below $T_{i}$ the contribution $\Delta v=$ $A|<Q>|^{2}$ was observed, similarly as in the other materials with incommensurate phase transitions [7, 8]. Therefore, from the temperature dependence of shear ultrasonic velocity the critical exponent for order parameter can be derived. The least square fits showed that $\Delta v \sim\left(T_{i}-T\right)^{2 \beta}$, with $\beta=0.345 \pm 0.005$ (see the inset of Fig.2). The value of $\beta$ is in a good agreement with the 3D X - Y model [9] and confirms NMR measurements of $\beta$ in BCCD crystals [10]. 
We should note, that in BCCD crystals small anomalies of longitudinal and shear ultrasonic velocity have been observed at other phase transitions of incomplete "devils staircase" sequence [11].

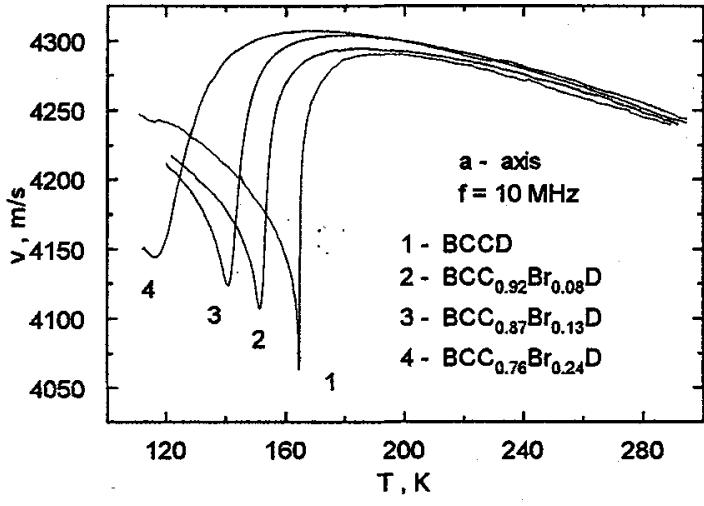

Figure 3: The temperature dependencies of longitudinal ultrasonic velocity in BCCD crystals doped with $\mathrm{Br}$.

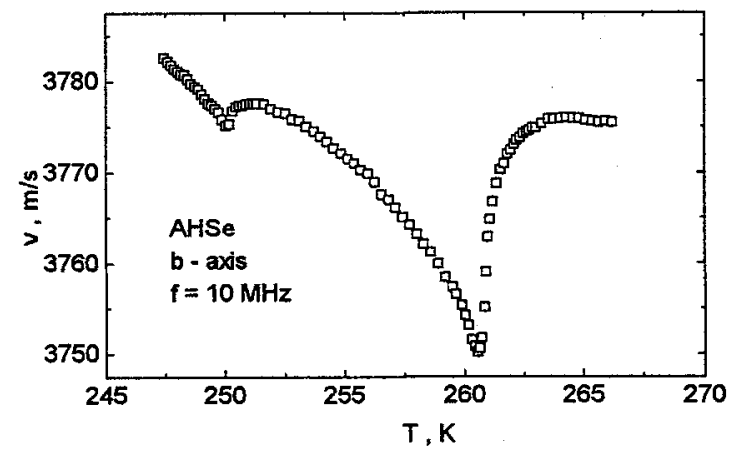

Figure 4: The temperature dependence of ultrasonic velocity measured along $b$ axis in AHSe single crystal.

The preliminary results of the influence of defects on the elastic anomalies in BCCD crystals doped with $\mathrm{Br}$ are shown in Fig.3. The longitudinal ultrasonic velocity anomalies at $T_{i}$ are shifted to lower temperatures and become broader. The shift of the phase transition can be explained by a widening of the unit cell due to incorporation of the larger $\mathrm{Br}^{-}$ions at $\mathrm{Cl}^{-}$sites (negative chemical pressure). Also the $v=f(\mathrm{~T})$ visualise defect induced phenomena like a decrease of velocity dip at $T_{i}$ and aconsiderable broadening of the elastic anomalies. This coincides with dielectric measurements [12]. More detail analysis and discussion of these defect induced phenomena will be presented elsewhere.

The incommensurate phase in AHSe crystals covers the temperature range 250-261 K [13]. From the measurements of the longitudinal ultrasonic velocity along the $b$ axis two minima corresponding to these temperatures could be resolved ( Fig.4). The minimum of $v=f(\mathrm{~T})$ at $\mathrm{T}_{c}$ $=250.2 \mathrm{~K}$ is attributed to the phase transition to the ferroelectric phase. The anomaly at $T_{c}$ was dependent on the sample history; e.g., when the following temperature runs through $T_{c}$ were performed, the velocity minimum decreased and shifted noticeably to lower temperatures below $T_{c}$. Such behaviour we attribute to the different domain state in the ferroelectric phase [5].

The measurements of frequency dependencies of elastic behaviour in the IC phase were reproducible as long the temperature was not lowered below $T_{c}$. Near the transition from $\mathrm{N}$ to IC phase at $\mathrm{T}_{\mathrm{i}}=260.6 \mathrm{~K}$ the ultrasonic velocity dispersion has been observed (Fig.5a). The ultrasonic attenuation maxima (Fig.5b)
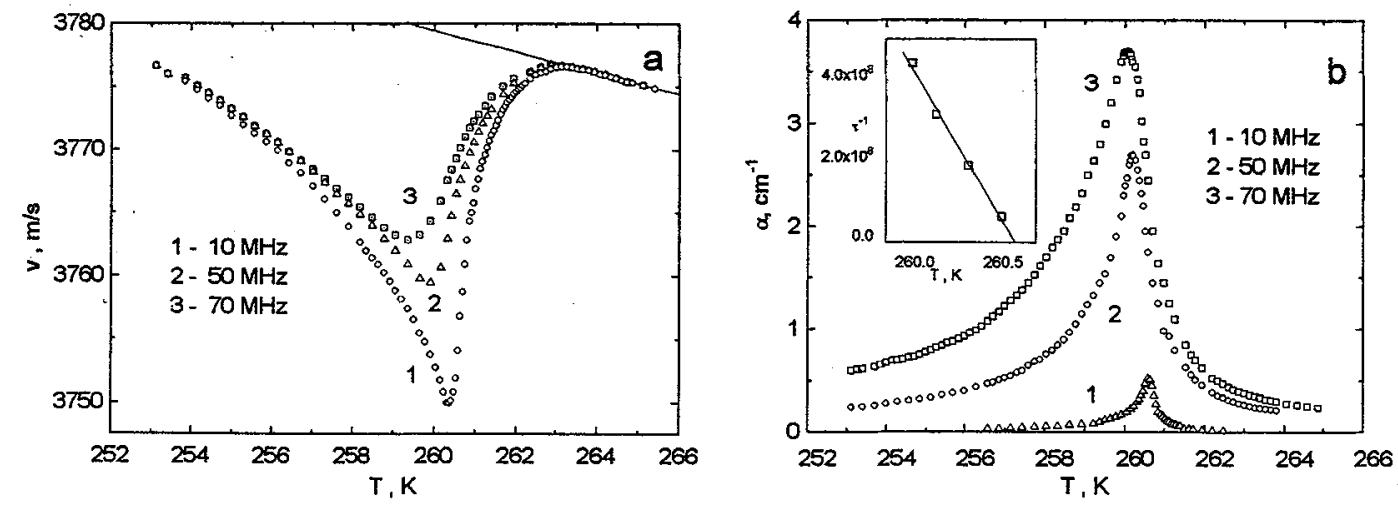

Figure 5: The temperature dependencies of longitudinal ultrasonic velocity (a) and attenuation coefficient (b) measured along $b$ - axis of AHSe crystal at frequencies $(\mathrm{MHz}): 1-10,2-50,3-70$. In the inset: the temperature dependence of inverse relaxation time. 
shifted to lower temperatures with increasing frequency, as it should be according to Eq.(1), assuming the relaxation time increases near $T_{i}$. The maxima of $\alpha$ appears when $\omega \tau=1$. From the positions of $\alpha$ peaks in temperature scale we were able to calculate temperature dependence of $\tau^{-1}$. A linear dependence of $\tau^{-1}=$ $f(\mathrm{~T})$ is obtained (see the inset of Fig. $5 \mathrm{~b}$ ). From this dependence, the amplitudon relaxation time in IC phase of AHSe crystal can be calculated: $\tau=5.1 \cdot 10^{-12} \mathrm{~T} /\left(\mathrm{T}_{\mathrm{i}}-\mathrm{T}\right)$ and it is considerably longer than in $\mathrm{BCCD}$ below $\mathrm{T}_{\mathrm{i}}=164 \mathrm{~K}$.

\section{CONCLUSIONS}

The temperature and frequency dependencies of the ultrasonic velocities and attenuation coefficients have been studied in the vicinity of the incommensurate phase transitions of BCCD and AHSe crystals. The major contribution to the longitudinal ultrasonic anomalies is due to the coupling to the amplitudon, whose relaxation time $\tau$ diverges as the transition temperature $T_{i}$ is approached with the temperature dependence given by: $\tau=\tau_{0} T_{i} /\left(T-T_{i}\right)$. The amplitudon relaxation time for AHSe $\left(\tau_{0}=5.1 \cdot 10^{-12} \mathrm{~s}\right)$ and $B C C D\left(\tau_{0}=1.3\right.$. $\left.10^{-13} \mathrm{~s}\right)$ crystals has been calculated from the ultrasound attenuation data. From the temperature dependence of shear ultrasonic wave velocity in IC phase below $T_{i}=164 \mathrm{~K}$ the critical exponent of order parameter $\left(\beta=0.345 \pm 0.005\right.$ ) has been determined for $\mathrm{BCCD}$. The substitution of $\mathrm{Cl}$ to $\mathrm{Br}$ in $\mathrm{BCC}_{1-x} \mathrm{Br}_{\mathrm{x}} \mathrm{D}$ crystals with $x<0.24$ manifest itself in broadening of elastic anomalies and shifting $T_{i}$ to lower temperatures with increase of $\mathrm{Br}$ contents.

\section{Acknowledgements}

The authors are grateful to G. Schaack and M. le Maire for providing Br doped BCCD crystals, to Z.Czapla for supplying AHSe crystals. Measurements were performed with equipment donated by the Alexander von Humbolt Stiftung. Special thanks to ICIFUAS - 11 Organising Committee for the financial support which enabled participation in the conference.

\section{References}

[1] Ting Wu, Fossheim K.,Wada T., Yaegashi Y., Yamauchi H. and Tanaka S., Phys. Rev. B47 (1993) $12197-12204$.

[2] Sorge G. and Straube V., Journal of Alloys and Compounds 211/212 (1994) 185 - 189.

[3] Valevichius V., Samulionis V. and Banys J., Journal of Alloys and Compounds 211/212 (1994) 369 373.

[4] Kezionis A., Orliukas A., Paulavicius K. and Samulionis V. , Mater. Sci. Forum 76 (1991) 229- 233.

[5] Valevicius V., Samulionis V., Banys J., Grigas J. and Yagi T., Ferroelectrics 156 (1994) 365 - 370.

[6] Kityk A.V., Sopruniuk V.P., Vlokh O.G., Sveleba S.A. and Czapla Z., J. Phys.: Condens. Matter 5 (1993) $7415-7424$.

[7] Kubinec P., Birks E., Schranz W. and Fuith A., Phys. Rev. B49 (1994) 6515 - 6523

[8] Rehwald W., Vonlanthen A., Kruger J.K., Wallerius R. and Unruh H.-G., J. Phys. C: Solid St. Phys. 13 (1980) $3823-3833$.

[9] Cowley R.A. and Bruce A.D., J. Phys. C 11 (1978) 3577 > 3584.

[10] Holzer K.-P., Petersson J., Hacker U.and Michel D., Ferroelectrics (1996) to be published.

[11] Unruh H.-G., Hero F. and Dvorak V., Solid St. Communs 70 (1989) 403 - 407.

[12] Le Maire M., Lopez Ayala A., Schaack G., Klopperpieper A. and Metz H., Ferroelectics 155 (1994) 335 - 340 .

[13] Czapla Z., Wiadomosci chemiczne 46 (1992) $55-67$. 